\title{
Ipriflavone promotes proliferation and osteogenic differentiation of periodontal ligament cells by activating GPR30/PI3K/AKT signaling pathway
}

This article was published in the following Dove Press journal:

Drug Design, Development and Therapy

\author{
Yuanyuan Han ${ }^{1,2}$ \\ Xuxia Wang ${ }^{2,3}$ \\ Dan Ma ${ }^{1,2}$ \\ Xiaoxiao $\mathrm{Wu}^{1,2}$ \\ Panpan Yang ${ }^{1,2}$ \\ Jun Zhang ${ }^{1,2}$
}

'Department of Orthodontics, Faculty of Stomatology, Shandong University, Jinan, ${ }^{2}$ Shandong Provincial Key Laboratory of Oral Tissue

Regeneration, School of Stomatology, Shandong University, Jinan,

${ }^{3}$ Department of Oral and Maxillofacial Surgery, Faculty of Stomatology,

Shandong University, Jinan, China
Correspondence: Jun Zhang School of Stomatology, Shandong University, No 44-I, Wenhua Xi Road, Jinan, 2500I2, Shandong, China Email zhangj@sdu.edu.cn
Objectives: This study was performed to investigate the effects and mechanism of ipriflavone (IP) on the proliferation and osteoblastic differentiation of periodontal ligament cells in vitro and periodontal tissue remodeling following orthodontic tooth movement (OTM) in vivo.

Materials and methods: Human periodontal ligament cells (hPDLCs) were cultured in vitro and cell counting kit-8, alkaline phosphatase (ALP) activity assay, plate clone formation assay, and alizarin red staining were used to test proliferation and osteogenic differentiation of hPDLCs. What is more, the expression of ALP, Runx2, and GPR30 was examined by real-time polymerase chain reaction and Western blot. To find out if PI3K/AKT signaling pathway was involved in the process, AKT and p-AKT were examined by Western blot. LY294002 (PI3K signaling pathway inhibitor) and small interfering RNA targeting GPR30 mRNA (siGPR30) were used to verify the function of GPR30-mediated PI3K/AKT pathway in this process. Twenty-four male Wistar rats were randomized into 2 groups, the control group with force application and the IP group with force application plus IP. Morphological changes in the periodontal tissue between roots of teeth were investigated using hematoxylin and eosin (HE) staining and bone morphogenetic protein-2 was detected to assess bone remodeling by immunohistochemical staining.

Results: In vitro, $10^{-7} \mathrm{M}$ IP was selected significantly promoting proliferation, ALP activity, colony forming efficiency, and mineral deposition $(P<0.05)$ on hPDLCs. Gene expressions of $A L P, R u n x 2, G P R 30$, and $p$-AKT were all upregulated than the control group $(P<0.05)$. According to the mechanism, promotion of ALP and Runx2 interdicted by LY294002 and siGPR30 reduced the activation of PI3K/AKT signaling pathway. In addition, HE staining and immunohistochemical staining results showed that the IP group had more new bone formation in the periodontal tissue compared to the control group in vivo.

Conclusion: IP can promote the expression of ALP and Runx2 which was probably related to the GPR30-mediated PI3K/AKT signaling pathway. Moreover, IP coordination seemed to have the potential to prevent relapsing following OTM.

Keywords: ipriflavone, periodontal ligament cells, osteogenic differentiation, GPR30, PI3K/AKT

\section{Introduction}

The relapse of moved teeth after orthodontic treatment is always a problem demanding prompt solution in relation to the successful treatment goals for orthodontic doctors. The biological mechanism of orthodontic relapse seems to be the same as that of orthodontic tooth movement (OTM), and relapse occurs more rapidly in the lack of orthodontic forces. ${ }^{1}$ During the process of OTM, a series of activated signal transduction pathways act on periodontal tissue by orthodontic force application leading to the remolding 
of periodontal ligament (PDL) and alveolar bone. ${ }^{2}$ Alveolar bone and osteoclasts prefer to participate in the later stage of relapse, while PDL plays an important role in early relapse following OTM. ${ }^{3}$ Composed of dense connective tissue, the PDL surrounds the root of the tooth between the cementum and the alveolar bone. Furthermore, the PDL contains abundant periodontal ligament cells (PDLCs) that participate in alveolar bone remolding. ${ }^{4}$ PDLCs play an important role in PDL homeostasis and regeneration among various PDLC populations. OTM and relapse are both a process of tissue remolding including periodontal tissue remolding and the subsequent alveolar bone formation, so promoting periodontal tissue remolding can shorten the retention time and prevent relapse following OTM.

In order to suppress the undesirable tendency toward rapid relapse and shorten the holding time, orthodontic doctors put in great efforts, for instance, drug therapy. There have been many explorations and innovations using various systemically or locally administrated pharmacologic agents such as simvastatin, ${ }^{5,6}$ bisphosphonate, ${ }^{7}$ relaxin, ${ }^{8}$ and osteoprotegerin protein. ${ }^{9,10}$ Estrogens are considered regulators of cell proliferation, bone growth, energy metabolism, and inflammation, etc. ${ }^{11}$ Estrogen replacement therapy (ERT) plays an assignable role in the prevention and treatment of perimenopausal symptoms including osteoporosis in the clinic. ${ }^{12}$ Meanwhile, it has been reported that ERT has numerous side effects, such as vaginal bleeding, edema, and increased incidence of breast cancer, venous thrombosis, stroke, and cardiovascular events. ${ }^{13,14}$ Researches show that phytoestrogens such as isoflavone and herbal medicines such as icariine, genistin ${ }^{15}$ have been introduced to take the place of ERT owing to the characteristics of significant curative effect and less side effects. Ipriflavone (IP), 7-isopropoxy3-phenyl-4H-1-benzopy-ran-4-one, a genus of isoflavone, which can directly act on bone, has been recognized as an inhibitor of bone resorption, with growth promoting properties by oxidative phosphorylation but devoid of intrinsic estrogenic activity. ${ }^{16}$ It has both therapeutic effects of estrogen and calcitonin without their side reaction because of its physiological metabolic process in vivo.

The conception of how estrogens mediate cellular signaling, the variety of estrogen receptors, the signal transduction pathways involved, and the expression and physiological function in tissue and organs have become increasingly important for osteogenesis. ${ }^{17}$ In recent years, many studies have found that membrane estrogen receptors can mediate fast non-gene effects. Prossnitz indicated that GPR30 is a 7 transmembrane $\mathrm{G}$ protein-coupled receptor constituted by 375 amino acid residues and participates in not only estrogen-dependent kinase activation but also transcriptional responses. ${ }^{18}$

We aimed to investigate the effect of IP on the proliferation and osteogenic differentiation of PDLCs in vitro and relapse following OTM in vivo through establishing rodent relapse models. We hypothesized that IP could promote the proliferation and osteoblastic differentiation of PDLCs via the activating of GPR30 and relevant signaling pathways.

\section{Materials and methods Antibodies and reagents}

IP was purchased from TargetMol Inc (Boston, MA, USA). Rabbit alkaline phosphatase (ALP), Runx2, GPR30 antibody, bone morphogenetic protein (BMP)-2 antibody were obtained from Abcam Inc (Cambridge, UK). AKT and p-AKT antibody were sourced from Cell Signaling Technologies (Danvers, MA, USA). TRIzol, Reverse Transcriptase, and SYBR $^{\circledR}$ Premix Ex Taq ${ }^{\mathrm{TM}}$ were obtained from TakaRa Biotech (Tokyo, Japan). Lipo 2000 and small interfering RNA (siRNA) were supplied by Thermo Fisher Scientific (Waltham, MA, USA). LY294002 was obtained from MedChemExpress (NJ, USA).

\section{Cell culture}

Primary human periodontal ligament cells (hPDLCs) were extracted from premolars with no caries and periodontal disease that had been extracted from healthy patients who provided informed consent for orthodontic needs (donors' age: 10-18 years old). Our study was conducted after receiving approval from the ethics committee of School of Stomatology, Shandong University (no 20160502). Approval to use the extracted tooth and informed consent of the parents or legal guardians of the children were obtained. The teeth were placed in precooled phosphate-buffered saline (PBS) containing 20\% antibiotics solution $(5,000$ units $/ \mathrm{mL}$ penicillin and $50 \mathrm{Ag} / \mathrm{mL}$ streptomycin; Solarbio, Beijing, China) after removal. Then, the periodontal tissue in the middle of the tooth root was scraped under aseptic conditions after washing three times with PBS. The tissues blocks were arranged at the bottom of the culture bottle added with $\alpha$-minimum essential medium ( $\alpha$-MEM; Hyclone, Logan, UT, USA) containing 20\% (v/v) fetal bovine serum (FBS; Biological Industries, Israel) and 1\% (v/v) antibiotics solution at $37^{\circ} \mathrm{C}$ in a humidified atmosphere of $5 \% \mathrm{CO}_{2}$. The cells were rinsed with PBS and released with $0.25 \%$ trypsin-EDTA (Solarbio) solution and subcultured in $\alpha$-MEM containing $10 \%$ FBS and $1 \%$ antibiotics when the cell was covered with the bottom of the bottle. 


\section{Cell proliferation assay}

hPDLCs were cultured with 5,000 cells per well in 96-well plates and treated with $\alpha$-MEM with $10 \%$ FBS for 24 hours to adhere to the plates. After cell synchronization by $1 \%$ FBS for 24 hours, the medium was changed to $\alpha$-MEM supplemented with $1 \% \mathrm{FBS}$ and IP at concentrations of $0,10^{-9}, 10^{-8}, 10^{-7}$, $10^{-6}, 10^{-5} \mathrm{M}$ for 24,48 , and 72 hours. Cell counting kit-8 (CCK-8; Dojindo Laboratories, Kumamoto, Japan) was used to measure cell proliferation of hPDLCs. In other words, $\alpha$-MEM and CCK- 8 solution were recombined in a ratio of 9:1 and $100 \mu \mathrm{L}$ miscible liquids were added to each well and the plates were incubated for 3 hours at $37^{\circ} \mathrm{C}$. The absorbance was measured at $450 \mathrm{~nm}$ using the SPECTROstar ${ }^{\text {Nano }}$ microplate reader (BMG Labtech, Ortenberg, Germany).

\section{ALP activity assay}

ALP activity of hPDLCs was measured using the manufacturer's instructions (Nanjing Jiancheng Bioengineering Institute, Nanjing, China). Firstly, hPDLCs were seeded in 6-well plates at a density of $1 \times 10^{5}$ cells $/ \mathrm{mL}$ in an osteogenic inducing fluid $(\alpha$-MEM supplemented with $10 \%$ FBS, $10^{-8} \mathrm{~mol} / \mathrm{L}$ dexamethasone, $50 \mathrm{mg} / \mathrm{L}$ ascorbic acid, and $10 \mathrm{mmol} / \mathrm{L} \beta$-glycerophosphate) with concentration gradient of IP. The common 10\% FBS $\alpha$-MEM without mineralized solution was used for the blank control. After 7 and 14 days of induction, cells were washed with $1 \times$ PBS three times and scraped into $400 \mu \mathrm{L} 1 \%$ Triton X-100 after 30 minutes on ice. Then, the cells were sonicated and centrifuged at $12,000 \times g$ for 15 minutes at $4^{\circ} \mathrm{C}$. Afterward, $30 \mu \mathrm{L}$ supernatant, $50 \mu \mathrm{L}$ buffer solutions, and $50 \mu \mathrm{L}$ matrix liquid were mixed, and incubated for 15 minutes at $37^{\circ} \mathrm{C}$. Next, $150 \mu \mathrm{L}$ colored solution was added and transferred to a 96-well plate. The absorbance of the samples was measured at $520 \mathrm{~nm}$ wavelength with a spectrophotometer. Standard phenol solution and double distilled water were used to replace the supernatant for standard wells and negative control (NC) wells. ALP activity was calculated according to the concentration of phenol in the standard well and the protein concentration which was detected by bicinchoninic acid (BCA, Solarbio) method.

\section{Plate clone formation assay and alizarin red $\mathrm{S}$ staining}

For clone formation assay, hPDLCs were seeded in 6-well plates with 700 cells per well, and cultured for 10 days. Formed cell clones were fixed and stained with crystal violet after washing with PBS. For alizarin red S staining, hPDLCs were seeded in 6-well plates at a density of
$2 \times 10^{5}$ cells per well. After 3 weeks of induction with common $10 \%$ FBS $\alpha$-MEM (blank control group), osteogenic inductive medium (control group), or osteogenic inductive medium plus $10^{-7} \mathrm{M}$ IP (IP group), cells were washed with $1 \times \mathrm{PBS}$ thrice, fixed with $4 \%$ paraformaldehyde for 30 minutes, washed with $1 \times$ PBS thrice again, and stained with crystal violet and $2 \%$ alizarin red $\mathrm{S}(\mathrm{pH} 4.2)$ for 15 minutes at room temperature. Photographs were taken using a microscope under 100× magnification (Olympus Corporation, Tokyo, Japan) and analyzed by the software Image-Pro Plus 6.0 (Media Cybernetics Inc, Rockville, MD, USA).

\section{siRNA transfection}

siRNA targeting GPR30 mRNA (siGPR30) and siRNA-NC were synthesized by Thermo Fisher Scientific. RNA oligonucleotides $(200 \mathrm{nM})$ were transiently transfected into hPDLCs by Lipofectamine 2000 (Thermo Fisher Scientific) and the medium was replaced 6 hours after transfection. The sequences of siRNA targeting GPR30 were as follows: sense CCTCATTGAGGTGTTCAA (HSS104377) and TCCTGTGCACCTTCATGTCGCTCTT (HSS104378). The sequences of NC and positive control siRNA were provided by Thermo Fisher Scientific (art no 12935400 and 14750100).

\section{RNA extraction and real-time polymerase chain reaction (RT-PCR) analysis}

hPDLCs were seeded in 6-well plates at a density of $2 \times 10^{5}$ cells per plate for 48 hours. Then, the control group was cultured in regular culture medium and the experimental group in $\alpha$-MEM supplemented with $10 \%$ FBS and IP at a concentration of $10^{-7} \mathrm{M}$. After being cultured for 24 and 48 hours, total RNA from hPDLCs with different treatments was isolated using $1 \mathrm{~mL} /$ well TRIzol reagent (Thermo Fisher Scientific). According to the manufacturer's instructions, reverse transcriptase (TaKaRa Biotech) was used for cDNA synthesis. RT-PCR was performed using SYBR ${ }^{\circledR}$ Premix Ex $\mathrm{Taq}^{\mathrm{TM}}$ (TaKaRa) with Roche 480 and in triplicate for each RNA sample. Primer sequences for ALP, Runx2, GPR30, and GAPDH were as follows: ALP: 5'-ATGGGCCTGGCTA-3' and 5'-CTTGGAGAAGACGCCAVGT-3'; Runx2: 5'-TCCACACCATTAGGGACCATC-3' and 5'-TGCTAATG CTTCGTGTTTCCA-3'; GPR30： 5'-GGTGTGAG GAGCATCTGTTCTT-3' and 5'-CCTTGAAGT GAGCCTGGCATT-3'; GAPDH: 5'-AGGTCGG TGTGAACGGATTTG- $3^{\prime}$ and $5^{\prime}$-TGTAGACCATGTA GTTGAGGTCA-3'. The amount of mRNA was calculated with GAPDH serving as a control. 


\section{Western blot analysis}

The proteins were extracted from hPDLCs using radioimmunoprecipitation assay buffer with 1\% PMSF for 30 minutes on ice and centrifuged at $12,000 \times g$ for 15 minutes to collect the supernatant. By measuring the concentrations using BCA protein assay kit (Solarbio), the protein samples were mixed with $5 \times$ sodium dodecyl sulfate polyacrylamide gel electrophoresis (SDS-PAGE) loading buffer at $100^{\circ} \mathrm{C}$ for 10 minutes. Total proteins $(20 \mu \mathrm{g})$ were subjected to SDS-PAGE and then transferred onto PVDF membranes. The membranes were blocked and probed with primary antibodies that recognized ALP, Runx2, GPR30, GAPDH, AKT, p-AKT. Secondary antibodies were selected according to the species of origin of the primary antibodies. After incubation, luminescent signals were detected using an enhanced chemiluminescence kit (Biological Industries, Kibbutz BeitHaemek, Isreal) and chemical imaging system (Amersham Imager 600; GE Healthcare, Little Chalfont, UK).

\section{Experimental animals}

Twenty-four male Wistar rats (8-week-old, mean weight $180 \pm 20$ g, provided by the experimental animal center at Shandong University, Jinan, China) were raised in plastic cages set at $23^{\circ} \mathrm{C}$. The study was approved by the Institutional Animal Care and Use Committee of Shandong University, and was carried out in accordance with the National Institutes of Health Guidelines for the Use of Laboratory Animals.

The animal models were established based on the method proposed by King et al. ${ }^{19}$ After intraperitoneal anesthesia with chloral hydrate $(4 \%, 0.3 \mathrm{~mL} / 100 \mathrm{~g})$, the rats were ready for the application of orthodontic devices including a closed coil spring, stainless steel ligature wire (0.008 inch; Tomy, Tokyo, Japan), and resin (Charisma; Heraeus Kulzer, Germany). As shown in Figure 1, the left upper first molar was mesially moved by the stretched spring with a force of $100 \mathrm{~g}$. The orthodontic force devices were removed after 10 days. Then, the rats were randomly divided into two groups ( $\mathrm{n}=12$ each): a control group with tooth movement only and an IP group with tooth movement plus IP. IP was given to the rats from the last day of tooth movement through intragastric administration at $10 \mathrm{mg} \mathrm{kg} /$ day for 10 days. Meanwhile, rats in the control group received the same daily dose of normal saline during the same period. The distance between maxillary first molar lingual sulcus point and third molar in lingual groove point was measured to evaluate the relapse distance.

\section{Hematoxylin and eosin (HE) staining}

Rats were anesthetized and cardiac perfused with $4 \%$ paraformaldehyde solution; we dissected the maxilla and retained the maxillary first molar and the surrounding tissue. Then, the samples were immobilized in $4 \%$ paraformaldehyde solution for 24 hours at $4{ }^{\circ} \mathrm{C}$, rinsed in PBS overnight at $4{ }^{\circ} \mathrm{C}$, macerated in $10 \%$ EDTA solution (pH 7.4) for decalcification for a period of 12 weeks at $4{ }^{\circ} \mathrm{C}$, dehydrated in ascending concentrations of ethanol, and embedded in paraffin.

Tissues were obtained from the near to far direction, and the thickness was $4 \mu \mathrm{m}$ for histological analysis. The periodontal tissues surrounding the upper first molar were observed. For histological examination, the sections were stained with HE and morphological changes of the periodontal tissue in the pressure side and tension side of the maxillary first molar were observed under a light microscope (BX51; Olympus Corporation).

\section{Immunohistochemical staining of BMP-2}

Many studies have found that statins can stimulate new bone formation by increasing expression of the $B M P-2$ gene in bone cell both in vivo and in vitro. ${ }^{20,21}$ So, we focus on the BMP-2 expression of PDL to evaluate IP on the osteogenic differentiation of PDLCs in vivo. The tissue tablets were placed in a glass cylinder containing xylene solution for dewaxing, hydrated through alcohol gradients, and washed with PBS. Antigen was repaired by immersing in $0.1 \%(\mathrm{w} / \mathrm{v})$ trypsin at $37^{\circ} \mathrm{C}$ for 10 minutes. After treatment with $3 \% \mathrm{H}_{2} \mathrm{O}_{2}$ and normal goat serum to block the activity of endogenous catalase and nonspecific binding for 10 minutes, the sections were incubated with BMP-2 monoclonal antibody (1:200) at $4^{\circ} \mathrm{C}$ overnight. Each section was then incubated with biotin labeled goat anti-rabbit immunoglobulin $\mathrm{G}$ for 10 minutes at $37^{\circ} \mathrm{C}$ and streptavidin biotin peroxidase complex methods were employed. Counterstained with hematoxylin when positive expression was observed in 10 minutes under microscope. In the dyeing process of the NC group, PBS was used to replace the primary antibody. Three samples in each group were selected for observing the distribution and change pattern of immunohistochemical staining in the periodontal tissues under a light microscope. Moreover, five visual fields were randomly chosen in the compression side and tension side for analysis by Image-Pro Plus.

\section{Statistical analysis}

All data were expressed as mean \pm SD from at least three replicates for each experiment. $t$-test or one-way analysis of variance was used to test the significance between the experimental groups and the control group using SPSS 22.0 software (IBM Corporation, Armonk, NY, USA) and GraphPad prism 6 (GraphPad Software Inc, La Jolla, CA, USA). $P$-value $<0.05$ was considered statistically significant. 
A

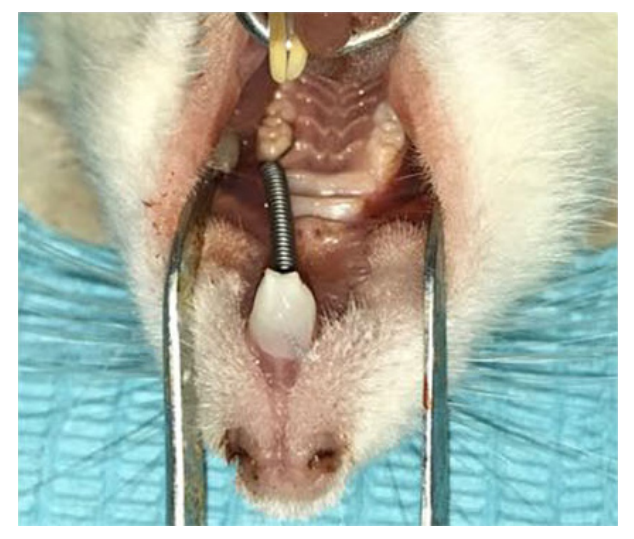

C
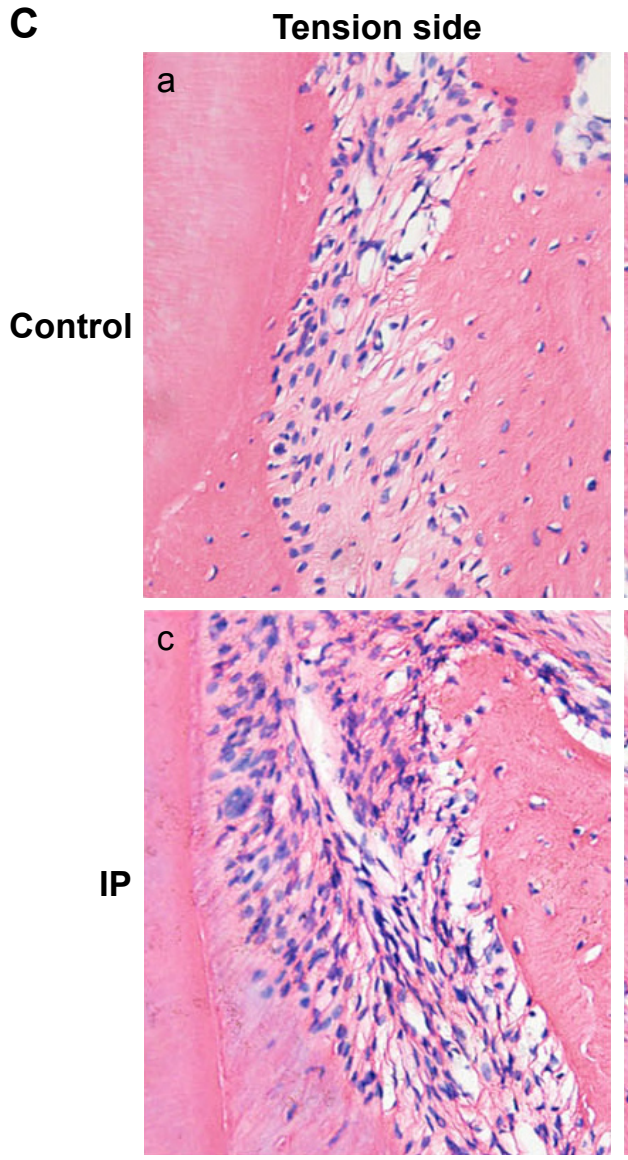

B

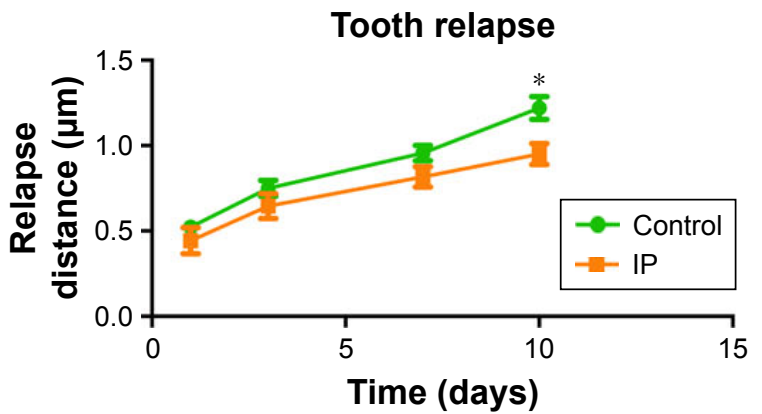

\section{Compression side}
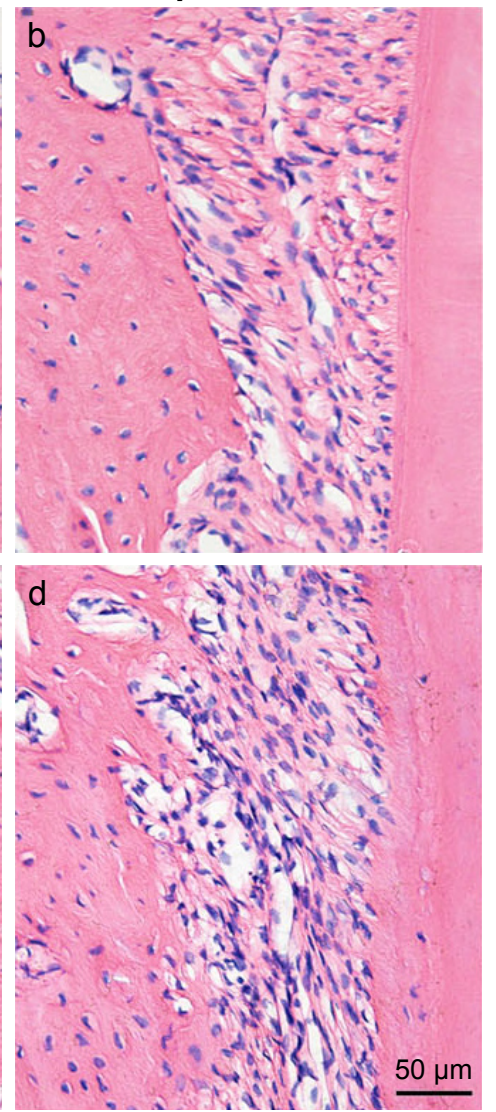

Figure I The animal model of relapse following orthodontic tooth movement (A). The relapse distance of control group and IP group (B). Histologic observation of the maxillary first molars on the compression side $(b, d)$ and tension side $(a, c)$. More new bone formation was observed in the IP group (c,d) than the control group $(a, b)$ (hematoxylin and eosin staining, magnification 200x) (C). Bars indicate $50 \mu \mathrm{m}$. $* P<0.05$.

Abbreviation: IP, ipriflavone.

\section{Results}

\section{The most effective concentration of}

\section{IP on cell proliferation and osteogenic differentiation of hPDLCs}

As indicated by CCK- 8 assays, results showed that at 24 hours after incubation, IP $\left(10^{-7}, 10^{-6}\right.$, and $\left.10^{-5} \mathrm{M}: P<0.01\right)$ promoted the proliferation of hPDLCs significantly with the control group (Figure 2). This promotion effect occurred dose dependently and reached its maximum at the concentration of $10^{-7} \mathrm{M}$. However, there is no significance between $10^{-8}$, $10^{-7}, 10^{-6}$, and $10^{-5} \mathrm{M}$ of IP on proliferation promotion. Moreover, there are statistically significant differences in cell proliferation between the control group and the IP group $\left(10^{-7} \mathrm{M}\right)$ (Figure 2). ALP activity has been widely used as a marker of early osteogenic differentiation of stem cells or progenitor cells. In our study, ALP activity was recorded on days 7 and 14 (Figure 2). On day 7, IP at the concentration 
A<smiles>CC(C)COc1ccc2c(=O)c(-c3ccccc3)coc2c1</smiles>

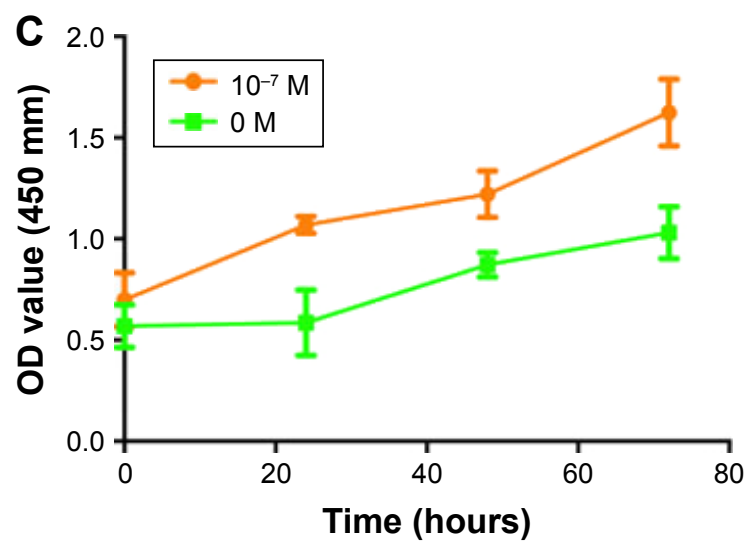

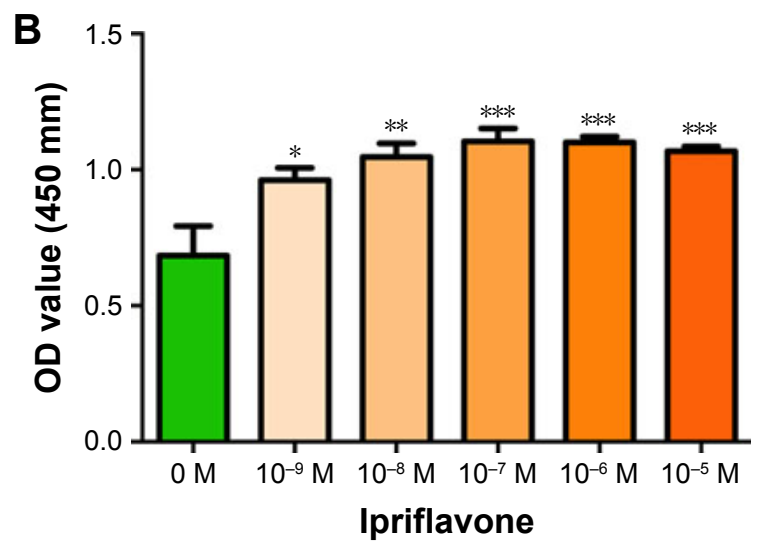
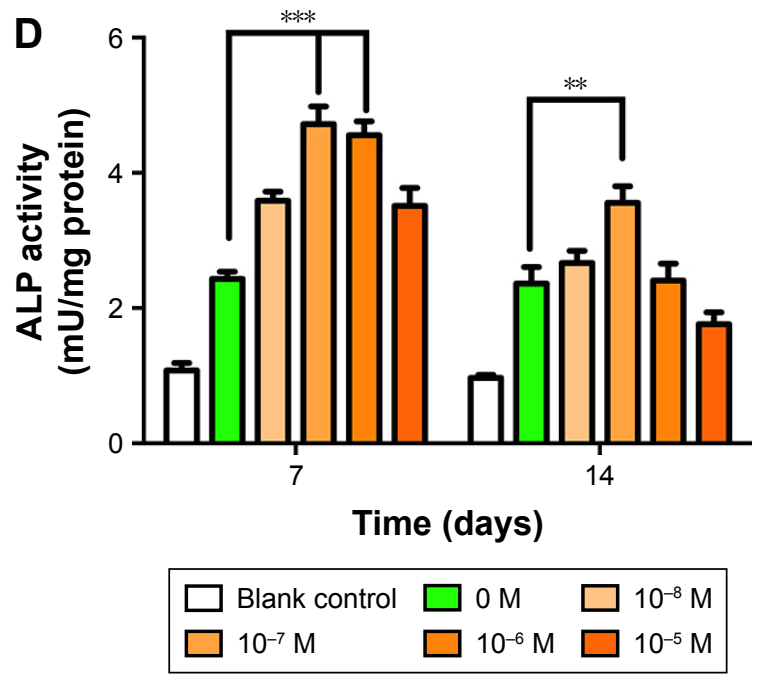

Figure 2 Chemical structural formula of IP. (A) Proliferative responses and osteogenic differentiation of hPDLCs stimulated with IP. (B) Proliferation of hPDLCs stimulated with IP for 24 hours by CCK-8 assay. (C) The growth curve of the control group (0 M) and the IP group (I0-7 M). (D) ALP activity quantification of hPDLCs stimulated with IP for 7 and I 4 days and blank control was used to prove the effect of the osteogenic inducing fluid. $* P<0.05, * * P<0.0$ I, $* * * P<0.00$ I versus control; mean \pm SD ( $\mathrm{n} \geq 3$ ).

Abbreviations: IP, ipriflavone; hPDLCs, human periodontal ligament cells; CCK-8, cell counting kit-8; ALP, alkaline phosphatase; SD, standard deviation; OD, optical density.

of $10^{-7}$ and $10^{-6} \mathrm{M}$ significantly promoted ALP activity compared with the positive control group $(P<0.05)$. But on day 14, ALP activity has no meaningful difference between different groups except $10^{-7} \mathrm{M}$ and the positive control. What is more, the blank control was used to prove the effect of the osteogenic inducing fluid. According to the CCK-8 and ALP results, we considered that IP can maximize the impact on hPDLCs at the concentration of $10^{-7} \mathrm{M}$. Thus, a concentration of $10^{-7} \mathrm{M}$ IP was adopted in the following studies.

\section{Effects of IP on colony formation rate and mineralization of hPDLCs}

Results of the clone formation assay are shown in Figure 3A and B. More and larger cell colonies were seen in the IP group after 10 days. For the mineralization assay, cells were cultured in osteogenic inducing medium with or without
$10^{-7} \mathrm{M}$ IP for 21 days. Culture dishes contained alizarin redstained nodules after culturing in the control and IP group. More and larger calcified nodules are seen in the IP group (Figure 3C). The effect of IP on calcified area fraction analysis is shown in Figure 3D. Mean $\pm \mathrm{SD}(\mathrm{n}=3) . * P<0.05$, $* * P<0.01$ versus control.

\section{Expression level of ALP, Runx2, GPR30, AKT, p-AKT of hPDLCs with IP}

In our research, ALP and Runx2 expression levels were detected by Western blot and RT-PCR to verify the osteogenic acceleration of IP on a molecular level. And the expression of GPR30, AKT, p-AKT was tested to find the mechanism of osteogenetic effects. Results showed that the expression levels of ALP, Runx2, GPR30 were improved by IP in both protein (Figure 4A) and mRNA (Figure 4B) level. 
A

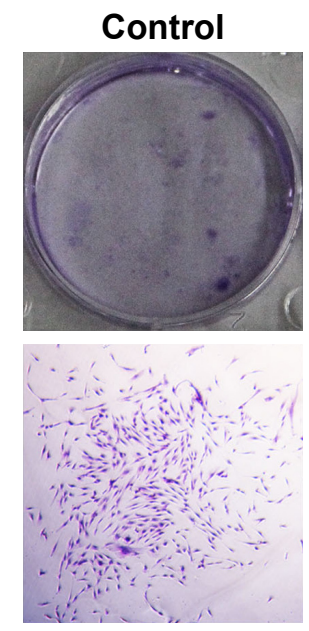

IP
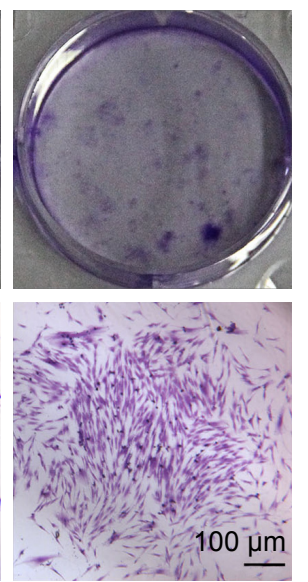

B

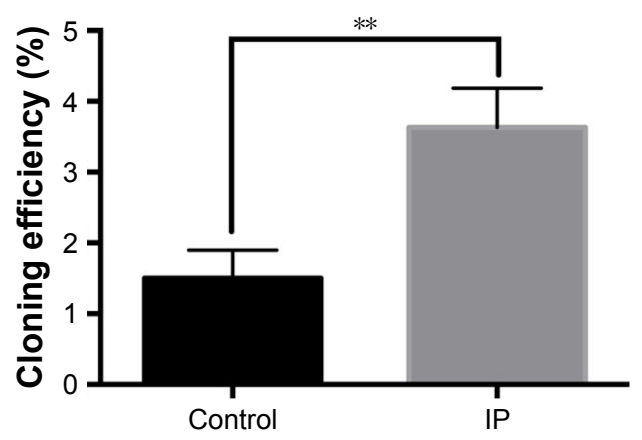

D

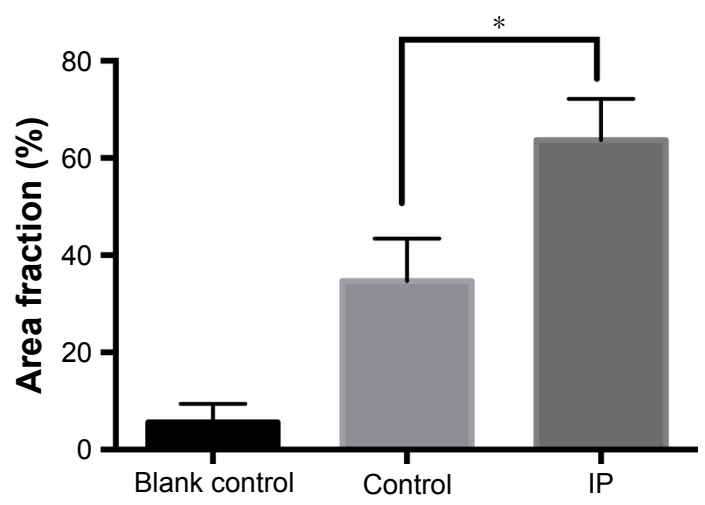

$100 \mu \mathrm{m}$
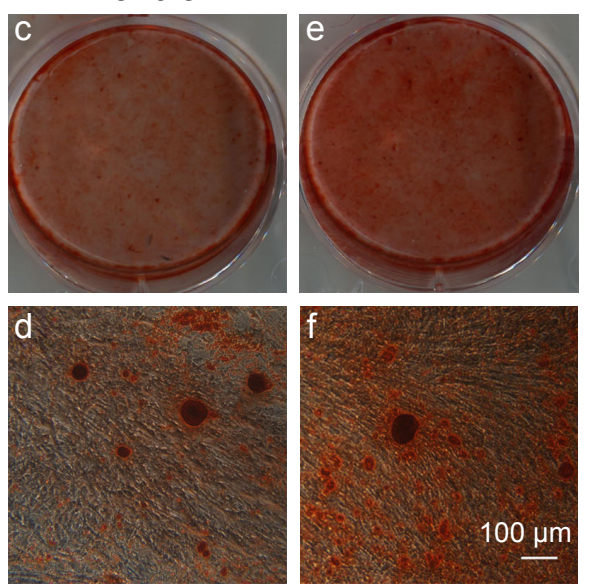

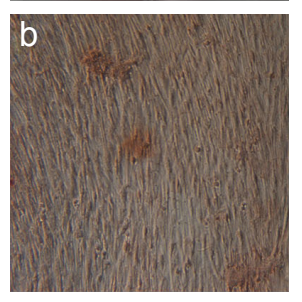

C Blank control

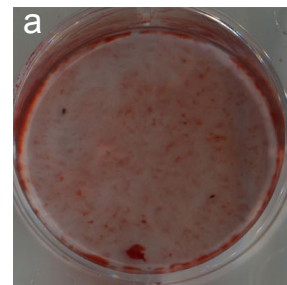

Figure 3 Effects of IP on colony formation rate $(\mathbf{A})$ and mineralization $(\mathbf{C})$ of hPDLCs. In the mineralization assay, more and larger calcified nodules can be seen in the IP group $(e, f)$ compared with the control group $(c, d)$. And the blank control group $(a, b)$ was used to prove the osteogenic potential of hPDLCs. IP (I0 $0^{-7}$ M) could stimulate the cloning efficiency (B) and mineralization area fraction (D) of hPDLCs significantly. Mean $\pm S D(n=3)$. $* P<0.05$, $* * P<0.01$ versus control. Magnification $\times 200$. Abbreviations: IP, ipriflavone; hPDLCs, human periodontal ligament cells; SD, standard deviation.

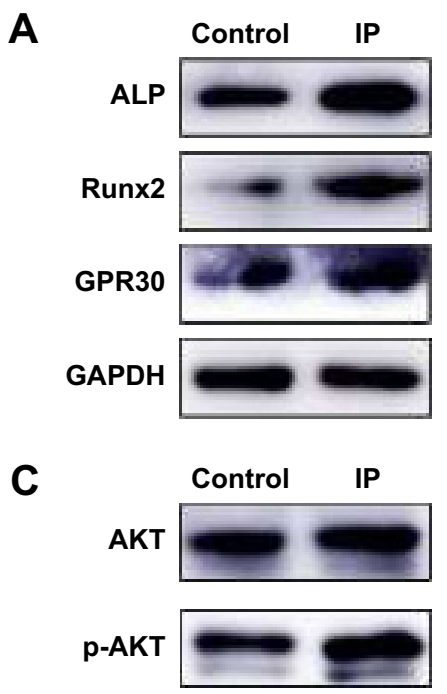

B

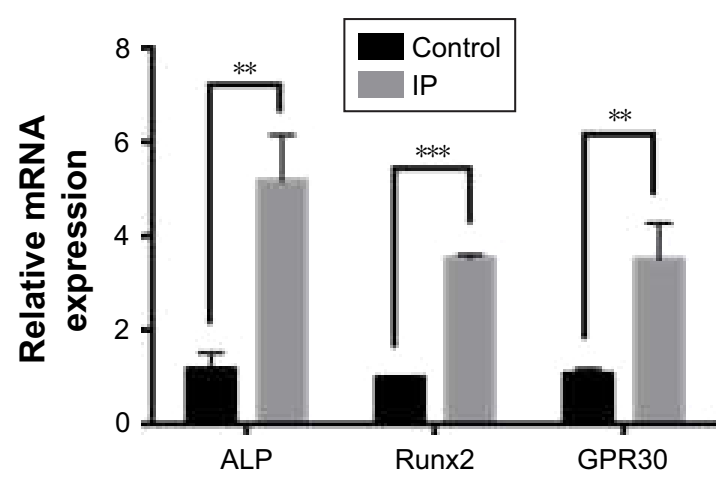

Figure 4 Expression of ALP, Runx2, GPR30 were all increased by IP in both protein level $(\mathbf{A})$ and mRNA level (B). The PI3K/AKT signaling pathway was activated by IP because the $\mathrm{P}$-AKT expression was high without the increase of total AKT (C). ${ }^{*} * P<0.0 \mathrm{I}$, $* * * P<0.00 \mathrm{I}$.

Abbreviations: IP, ipriflavone; ALP, alkaline phosphatase. 
Besides, the higher expression of p-AKT in invariant total AKT indicated that the PI3K/AKT signaling pathway was activated (Figure 4C).

\section{Osteogenic promotion of IP could be stopped by PI3K pathway inhibitor LY294002}

To characterize the protective signaling pathway, we used LY294002 (an inhibitor of PI3K signaling) in hPDLCs with IP. Then, the osteogenic genes ALP and Runx2 were reduced in both protein level and mRNA level (Figure 5). We conclude that IP promotes osteogenic differentiation via the PI3K/AKT signaling pathway.

\section{Contribution of GPR30 toward PI3K/AKT pathway activation}

hPDLCs were treated with siGPR30 and IP, and Western blot assays were performed to monitor expression levels of $\mathrm{AKT}$ and p-AKT. The fluorescence pictures were used to show the transfection efficiency (Figure 6A-C). We found that the activation of PI3K/AKT pathway was interdicted by
siGPR30 (Figure 6D and E), which indicated that GPR30 plays an important role in the process of activation of PI3K/AKT pathway.

\section{OTM relapse and HE staining}

There were no significant changes in the body weight of rats and no obvious swelling and inflammation during the experiment. The maxillary first molar mesial movement occurred, indicating that the orthodontic relapse model was established successfully (Figure 1A). Distance results of the first molar of the two groups increased with time and had similar movement curves; first, rapid movement occurred, and as time progressed, the relapse rate slowed down (Figure 1B). After removal of the device, the mesially moved upper first molars relapsed to the distal direction under the traction of the periodontal fibers. In contrast to the OTM, in the course of relapse, the distal PDL of the root is the pressure zone, and the mesial is the tension zone. Osteogenesis of periodontal tissue of the IP group was faster than that of the control group, and more alveolar bone formation was seen in the distal part (Figure 1C).
A

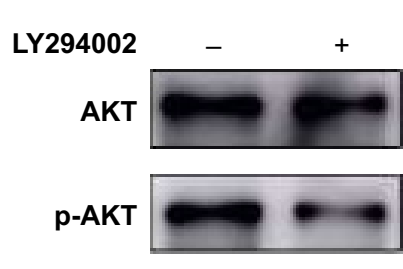

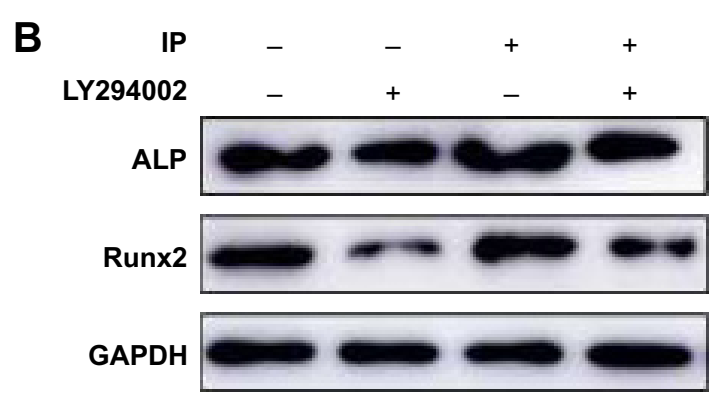

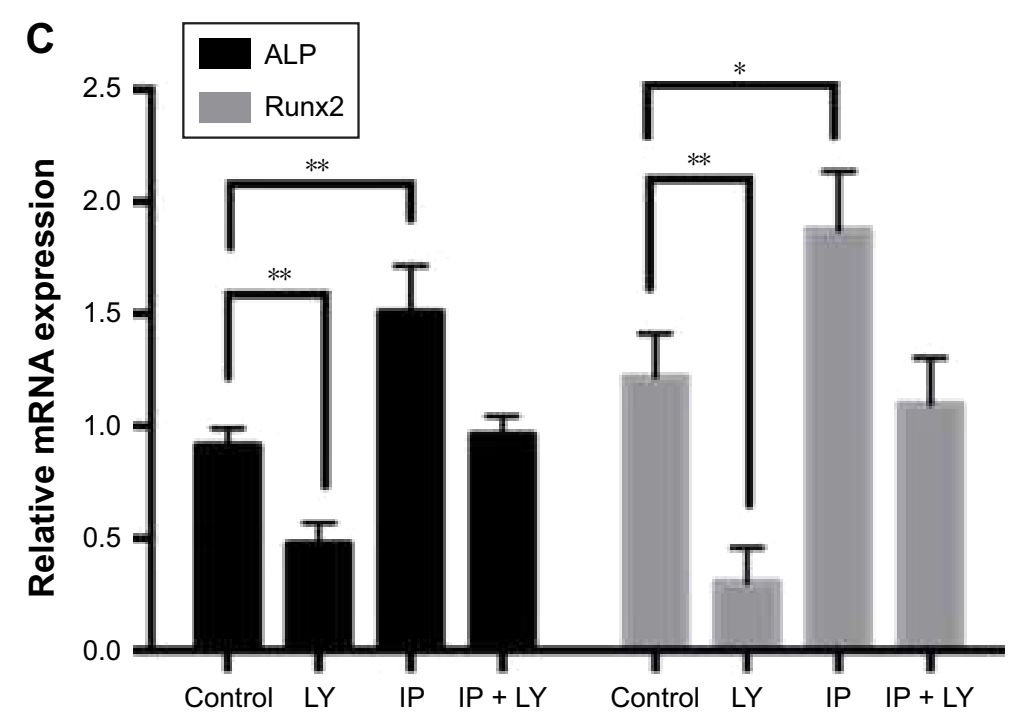

Figure 5 Effects of LY294002 on AKT and P-AKT expression of hPDLCs. (A) The increased expression of osteogenic marker proteins can be interdicted by PI3K pathway inhibitor LY294002 in hPDLCs. The expression level of ALP and Runx2 was tested by Western blot (B) and RT-PCR (C). $* P<0.05, * * P<0.01$.

Abbreviations: IP, ipriflavone; ALP, alkaline phosphatase; hPDLCs, human periodontal ligament cells; RT-PCR, real-time polymerase chain reaction; LY, LY294002. 
A
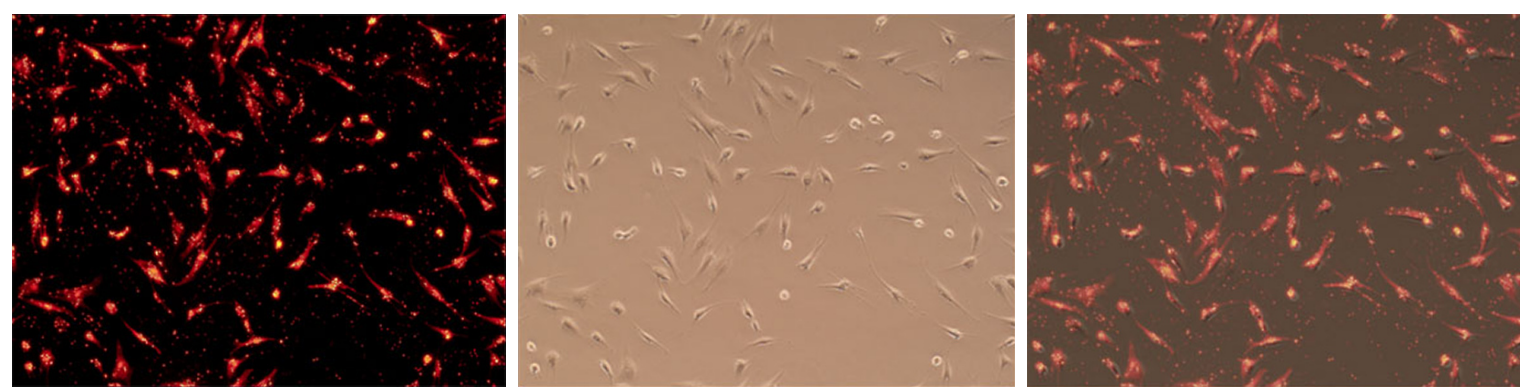

B

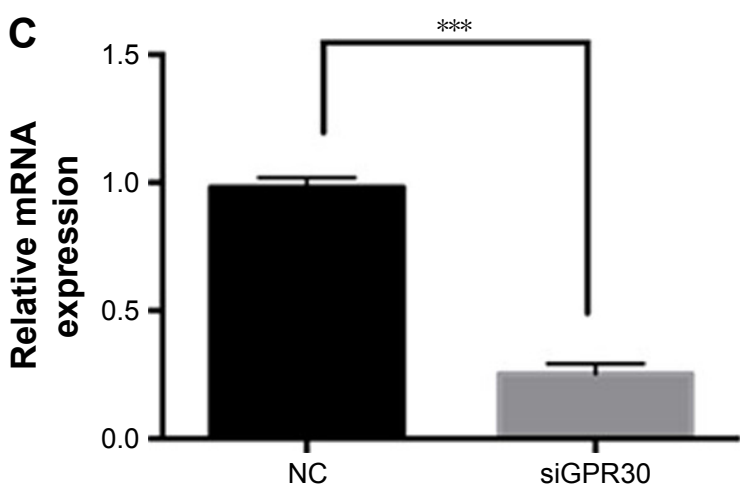

D

E
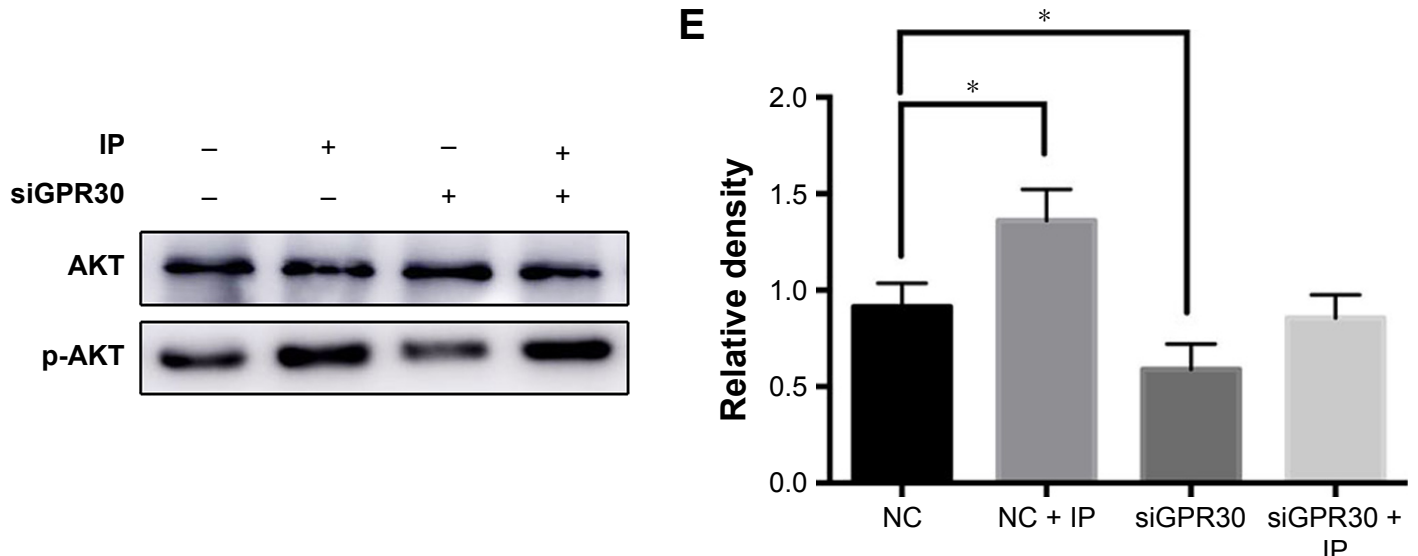

Figure 6 GPR30 knockdown eliminated IP-induced PI3K/AKT pathway activation of hPDLCs. The siRNA transfection efficiency of hPDLCs was tested by using the positive control with fluorescence. (A) Western blot (B) and PCR (C) results of GPR30 knockdown of hPDLCs. The expression of AKT and p-AKT was assessed by Western blot. (D) Quantitative analysis of Western blot band from (D) was performed. (E) $* P<0.05, * * * P<0.00$ I. Magnification $\times 200$.

Abbreviations: IP, ipriflavone; siRNA, small interfering RNA; hPDLCs, human periodontal ligament cells; PCR, polymerase chain reaction; NC, negative control; siGPR30, siRNA targeting GPR30 mRNA.

\section{Immunohistological analysis for BMP-2}

Immunohistochemical staining showed that the positive staining was caused by brown yellow granules and distributed in the cytoplasm and hematoxylin-stained nuclei were blue purple. The strong positive staining was a result of brown granules, and the weak positive staining pale yellow granules as shown in Figure 7. In the control group, low expression of BMP-2 was seen in the periodontal tissue; however, in the IP group, BMP-2 expression was detected in the osteoblasts, hPDLCs inside the blood vessels, and fibroblasts in the connective tissue between the alveolar bone and cementum.

\section{Discussion}

Long-term stability of OTM needs the coordination of appropriate tissue remodeling, growth development, good treatment result, and a suitable retention protocol. ${ }^{22}$ The essence of OTM is a process in which malposed teeth move to the normal but not stable position, and the promotion of restoration of normal periodontal tissue is essential to stabilize the teeth in the alveolar cavity. This investigation therefore examined the effects of IP on the relapse potential after OTM, with particular attention to proliferation, osteogenic differentiation, and GPR30-induced signaling pathway of hPDLCs. 
A
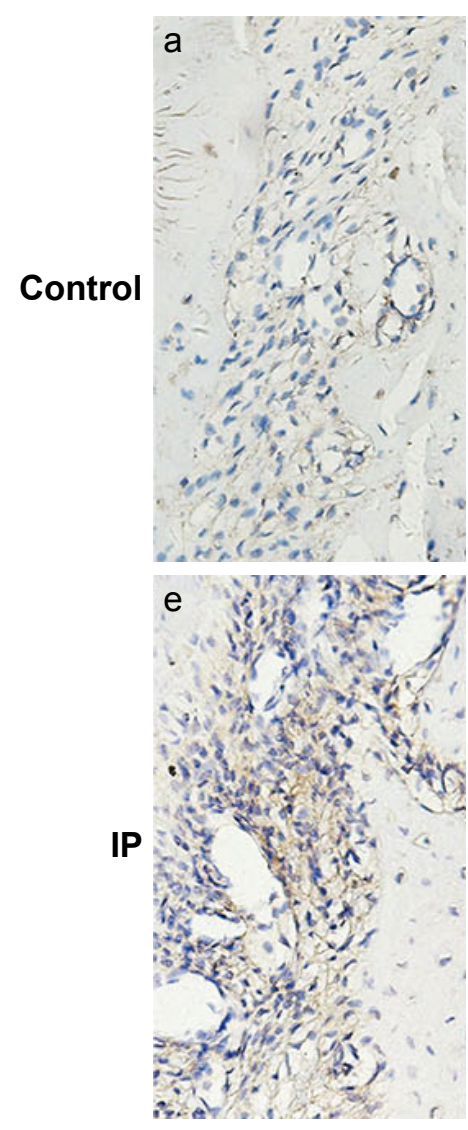

Tension side
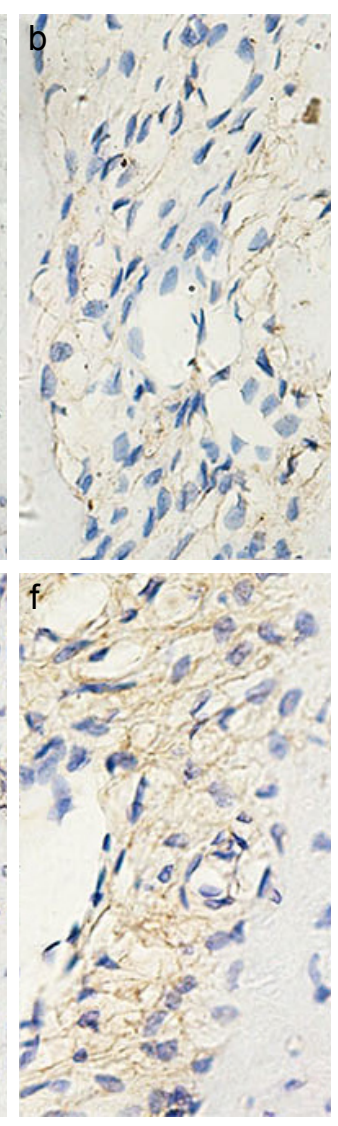

Compression side
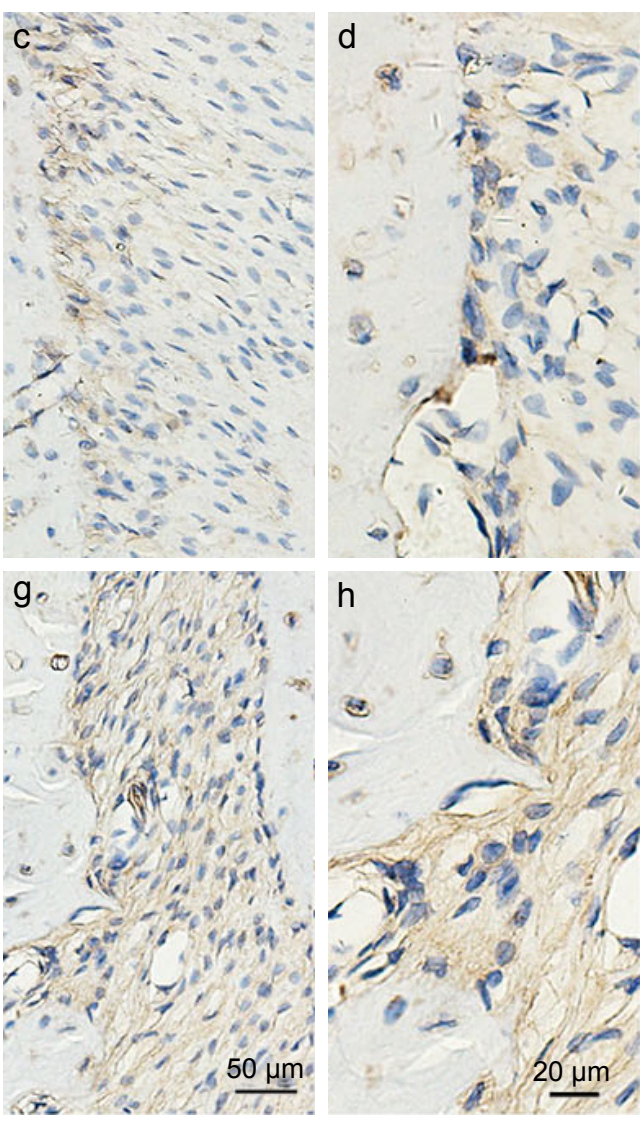

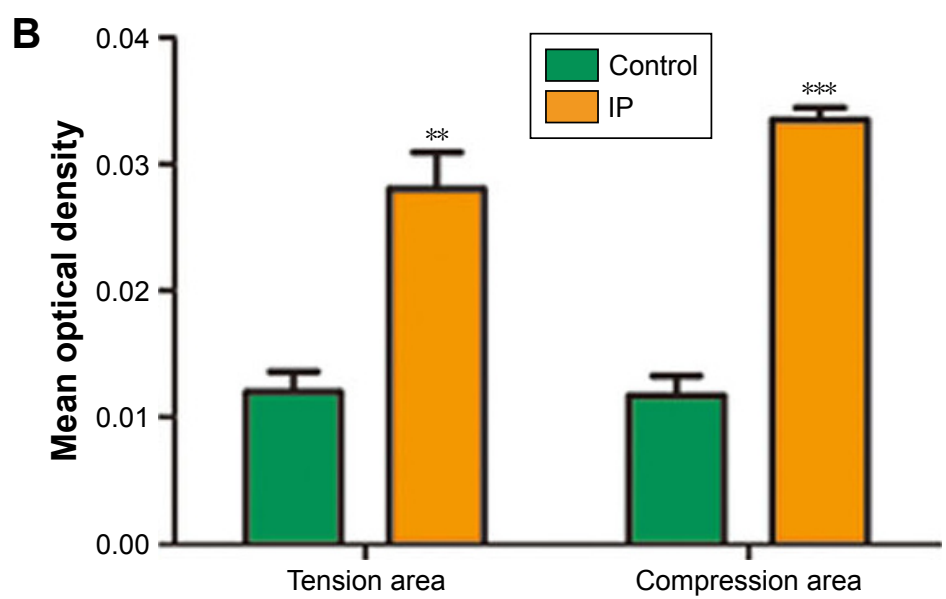

Figure 7 (A) Immunohistochemical staining of BMP-2. (a, c, e, g were captured in $\times 200$, and b, d, f, h were captured in $\times 400$. The expression of BMP-2 in the IP group was more than the control group. Bars indicate $20 \mu \mathrm{m}$. (B) Data analysis results of the immunohistochemical staining of $\mathrm{BMP}-2$. $* * P<0.01, * * * P<0.00 \mathrm{I}$. Abbreviations: IP, ipriflavone; BMP, bone morphogenetic protein.

IP is classified as one of the isoflavones in phytoestrogens, which exist in the food of humans and animals, and has been proposed as a potential agent for treating and preventing cancer, cardiovascular disease, ${ }^{23}$ and osteoporosis. ${ }^{24}$ This drug has been suggested to have a positive effect on bone mineral density in postmenopausal women. ${ }^{25}$ IP has a primary role in the therapy of metabolic bone diseases probably because of the preventable action against bone resorption ${ }^{26}$ and the stimulation on bone formation ${ }^{27}$ due to the direct effect on osteoclasts and osteoblasts. Ingesting IP daily before or after guided bone regeneration inhibits the resorption of augmented tissue and would be helpful in improving the quality of newly generated bone beyond the skeletal envelope. ${ }^{28}$ The main reason for the recurrence of OTM is 
the resilience of periodontal fibers. Therefore, the inhibition of alveolar resorption and bone formation on the pressure side play a key role in increasing the stability of the teeth. HE staining shows that in the IP group osteoclasts and a small amount of new bone can be seen at the same time at the edge of alveolar bone indicating that bone resorption and repair coexist at this stage and the relapse rate continues to slow down. BMP-2 is one of the most effective cytokines to promote bone growth, which induces the differentiation of mesenchymal stem cells into osteoblasts. It is produced by osteoblasts and exists in many bone and bone-like tissues. Our results showed that IP could expedite the process of bone remodeling of periodontal tissues, especially on the pressure side, so that its function can be extended in orthodontics.

During the process of osteogenic differentiation, two distinguishable events occur: proliferation and osteogenic differentiation of bone forming cells, which eventually leads to extracellular matrix maturation and mineralization. ${ }^{29}$ Unlike estrogen, IP has no side effects which suggests that the effects of IP on the PDL tissue are different from those of estrogens. In this regard, IP may act analogous to selective estrogen receptor modulators that are structurally analogous to isoflavones. It has been reported that genistein is the most abundant isoflavone in soybeans. According to Kakai et al's experiments, ${ }^{30}$ the dose-response curve showed a slight initial increase of pro-liberation up to $10^{-10} \mathrm{M}$ IP and a stimulating effect was apparent only above $10^{-9} \mathrm{M}$ IP, eliciting a maximal effect at $10^{-7} \mathrm{M}$ on ALP activity.

In vitro studies first showed the presence of estrogen receptors and gene responses to estrogen in osteoblasts rather than in osteoclasts. ${ }^{31}$ Jönsson et al investigated the $\mathrm{ER} \alpha$ and ER $\beta$ protein expression in hPDLCs derived from both male and female subjects by immunocytochemistry showing ER $\beta$ but not ER $\alpha$ immunoreactivity in these cells. ${ }^{32}$ Luo et al demonstrated for the first time that GPR30 was expressed in hPDLCs. ${ }^{33}$ The PI3K/AKT signaling pathway plays an important role in bone tissue not only accelerating the proliferation and differentiation but inhibiting apoptosis in osteoblasts. ${ }^{34,35}$ Activation of GPR30 could mediate many rapid cellular signaling events such as ERK pathway, PI3K/AKT pathway, and $\mathrm{Ca}^{2+}$ mobilization. ${ }^{36}$ It has been proved that upregulated GPR30 expression can activate the downstream PI3K/AKT signaling pathway with estrogen treatment in endometrial cancer cells. ${ }^{37}$

Bone remodeling that occurs during OTM is a biological process involving an acute inflammatory response in the periodontal tissues. Orthodontic forces induce a multifaceted bone remodeling response. ${ }^{38}$ In this in vitro study, hPDLCs were successfully isolated and we demonstrated that IP treatment enhanced the proliferation and osteoblastic differentiation of hPDLCs in vitro. Moreover, we confirmed that GPR30 plays an important role in the process of osteogenic differentiation. Collectively, our study demonstrates that IP promotes proliferation and osteoblastic differentiation of PDLCs after OTM through the GPR30/PI3K/AKT signaling pathway. To confirm the concrete function of IP and explore the underlying mechanisms, other signaling pathways need to be elucidated.

Our results suggested that IP can promote proliferation and osteogenic differentiation of PDLCs in vitro and periodontal reorganization following experimental tooth movement in vivo. Our data clearly show that the optimal concentration of IP is $10^{-7} \mathrm{M}$ and its function is dependent on GPR30, which could activate downstream PI3K/AKT signaling pathway. All these findings indicate that IP could represent a potential therapeutic tool to prevent relapse following OTM.

\section{Acknowledgments}

This work was supported by the National Natural Science Foundation of China (no 81371180) and the Science and Technology Development Program of Shandong Province (2014GGH218024).

\section{Disclosure}

The authors report no conflicts of interest in this work.

\section{References}

1. Franzen TJ, Brudvik P, Vandevska-Radunovic V. Periodontal tissue reaction during orthodontic relapse in rat molars. Eur J Orthod. 2013; 35(2): 152 .

2. Krishnan V, Davidovitch ZE. Cellular, molecular, and tissue-level reactions to orthodontic force. Am J Orthod Dentofacial Orthop. 2006; 129(4):469.

3. Feng L, Yang R, Liu D, et al. PDL Progenitor-mediated PDL recovery contributes to orthodontic relapse. J Dent Res. 2016;95(9):1049-1056.

4. Somerman M, Young MF, Foster RA, Moehring JM, Imm G, Sauk JJ. Characteristics of human periodontal ligament cells in vitro. Arch Oral Biol. 1990;35(3):241-247.

5. Han G, Chen Y, Hou J, et al. Effects of simvastatin on relapse and remodeling of periodontal tissues after tooth movement in rats. Am J Orthod Dentofacial Orthop. 2010;138(5):550.

6. Vieira GM, Chaves SB, Ferreira VM, Freitas KM, Amorim RF. The effect of simvastatin on relapse of tooth movement and bone mineral density in rats measured by a new method using microtomography. Acta Cir Bras. 2015;30(5):319.

7. Kim TW, Yoshida Y, Yokoya K, Sasaki T. An ultrastructural study of the effects of bisphosphonate administration on osteoclastic bone resorption during relapse of experimentally moved rat molars. Am J Orthod Dentofacial Orthop. 1999;115(6):645.

8. Hirate Y, Yamaguchi M, Kasai K. Effects of relaxin on relapse and periodontal tissue remodeling after experimental tooth movement in rats. Connect Tissue Res. 2012;53(3):207-219. 
9. Hudson JB, Hatch N, Hayami T, et al. Local delivery of recombinant osteoprotegerin enhances postorthodontic tooth stability. Calcif Tissue Int. 2012;90(4):330-342.

10. Schneider DA, Smith SM, Campbell C, Hayami T, Kapila S, Hatch NE. Locally limited inhibition of bone resorption and orthodontic relapse by recombinant osteoprotegerin protein. Orthod Craniofac Res. 2015; 18(S1):187-195.

11. Somjen D, Katzburg S, Sharon O, Grafi-Cohen M, Knoll E, Stern N. The effects of estrogen receptors $\alpha$ - and $\beta$-specific agonists and antagonists on cell proliferation and energy metabolism in human bone cell line. $J$ Cell Biochem. 2011;112(2):625-632.

12. Zheng $\mathrm{X}$, Lee $\mathrm{S}-\mathrm{K}$, Chun OK. Soy isoflavones and osteoporotic bone loss: a review with an emphasis on modulation of bone remodeling. J Med Food. 2016;19(1):1-14.

13. Nelson HD, Humphrey LL, Nygren P, Teutsch SM, Allan JD. Postmenopausal hormone replacement therapy: scientific review. JAMA. 2002; 288(7):872-881.

14. Schierbeck L. Primary prevention of cardiovascular disease with hormone replacement therapy. Climacteric. 2015;18(4):492-497.

15. Morabito N, Crisafulli A, Vergara C, et al. Effects of genistein and hormone-replacement therapy on bone loss in early postmenopausal women: a randomized double-blind placebo-controlled study. J Bone Miner Res. 2002;17(10):1904-1912.

16. Yamazaki I. Effect of ipriflavone on the response of uterus and thyroid to estrogen. Life Sci. 1986;38(8):757-764.

17. Barton M. Not lost in translation: emerging clinical importance of the G protein-coupled estrogen receptor GPER. Steroids. 2016;111: 37-45.

18. Prossnitz ER, Arterburn JB, Smith HO, Oprea TI, Sklar LA, Hathaway HJ. Estrogen signaling through the transmembrane $\mathrm{G}$ protein-coupled receptor GPR30. Annu Rev Physiol. 2008;70(1):165-190.

19. King GJ, Keeling SD, McCoy EA, Ward TH. Measuring dental drift and orthodontic tooth movement in response to various initial forces in adult rats. Am J Orthod Dentofacial Orthop. 1991;99(5):456-465.

20. Mundy G, Garrett R, Harris S, et al. Stimulation of bone formation in vitro and in rodents by statins. Science. 1999;286(5446):1946-1949.

21. Garrett IR, Mundy GR. The role of statins as potential targets for bone formation. Arthritis Res. 2002;4(4):237-240.

22. Franzen TJ. The influence of low-level laser on orthodontic relapse in rats. Eur J Orthod. 2015;37(1):111-1117.

23. Arliss RM, Biermann CA. Do soy isoflavones lower cholesterol, inhibit atherosclerosis, and play a role in cancer prevention? Holist Nurs Pract. 2002;16(5):40.

24. Kruger MC, Wolber FM. Osteoporosis: modern paradigms for last century's bones. Nutrients. 2016;8(6):376.
25. Melis GB, Paoletti AM, Cagnacci A, et al. Lack of any estrogenic effect of ipriflavone in postmenopausal women. J Endocrinol Invest. 1992;15(10):755-761.

26. Bonucci E, Ballanti P, Martelli A, et al. Ipriflavone inhibits osteoclast differentiation in parathyroid transplanted parietal bone of rats. Calcif Tissue Int. 1992;50(4):314-319.

27. Benvenuti S, Tanini A, Frediani U, et al. Effects of ipriflavone and its metabolites on a clonal osteoblastic cell line. J Bone Miner Res. 1991; 6(9):987.

28. Ito K, Minegishi T, Takayama T, Tamura T, Yamada Y, Sato S. Effects of ipriflavone on augmented bone using a guided bone regeneration procedure. Clin Oral Implants Res. 2007;18(1):60-68.

29. Kärner E, Backesjo CM, Cedarvall J, Sugars RV, Ahrlund-Richter L, Wendel M. Dynamics of gene expression during bone matrix formation in osteogenic cultures derived from human embryonic stem cells in vitro. Biochim Biophys Acta. 2008;1790(2):110-118.

30. Kakai Y, Kawase T, Nakano T, Mikuni-Takagaki Y, Saito S. Effect of ipriflavone and estrogen on the differentiation and proliferation of osteogenic cells. Calcif Tissue Int. 1992;51(1):S11-S15.

31. Eriksen EF, Colvard DS, Berg NJ, et al. Evidence of estrogen receptors in normal human osteoblast-like cells. Science. 1988;241(4861): 84-86.

32. Jönsson D, Andersson G, Ekblad E, Liang M, Bratthall G, Nilsson BO. Immunocytochemical demonstration of estrogen receptor $\beta$ in human periodontal ligament cells. Arch Oral Biol. 2004;49(1):85-88.

33. Luo LJ, Liu F, Lin ZK, et al. Genistein regulates the IL-1 beta induced activation of MAPKs in human periodontal ligament cells through G protein-coupled receptor 30. Arch Biochem Biophys. 2012; 522(1):9-16.

34. Guntur AR, Rosen CJ. The skeleton: a multi-functional complex organ: new insights into osteoblasts and their role in bone formation: the central role of PI3Kinase. J Endocrinol. 2011;211(2):123-130.

35. Ling L, Dombrowski C, Foong KM, et al. Synergism between Wnt3a and heparin enhances osteogenesis via a phosphoinositide 3-kinase/Akt/RUNX2 pathway. J Biol Chem. 2010;285(34): 26233-26244.

36. Prossnitz ER. GPER modulators: opportunity Nox on the heels of a class Akt. J Steroid Biochem Mol Biol. Epub 2017 Mar 8.

37. Wei Y, Zhang Z, Liao H, et al. Nuclear estrogen receptor-mediated Notch signaling and GPR30-mediated PI3K/AKT signaling in the regulation of endometrial cancer cell proliferation. Oncol Rep. 2012; 27(2):504-510.

38. Meeran NA. Cellular response within the periodontal ligament on application of orthodontic forces. J Indian Soc Periodontol. 2013; 17(1):16-20.
Drug Design, Development and Therapy

\section{Publish your work in this journal}

Drug Design, Development and Therapy is an international, peerreviewed open-access journal that spans the spectrum of drug design and development through to clinical applications. Clinical outcomes, patient safety, and programs for the development and effective, safe, and sustained use of medicines are the features of the journal, which
Dovepress

has also been accepted for indexing on PubMed Central. The manuscript management system is completely online and includes a very quick and fair peer-review system, which is all easy to use. Visit http://www.dovepress.com/testimonials.php to read real quotes from published authors. 